\title{
Haptic-Guided Teleoperation of a 7-DoF Collaborative Robot Arm with an Identical Twin Master
}

\author{
Jayant Singh ${ }^{1}$, Aravinda Ramakrishnan Srinivasan ${ }^{1}$, Gerhard Neumann ${ }^{1,2}$ and Ayse Kucukyilmaz ${ }^{1,3}$
}

\begin{abstract}
In this study, we describe two techniques to enable hapticguided teleoperation using 7-DoF cobot arms as master and slave devices. A shortcoming of using cobots as master-slave systems is the lack of force feedback at the master side. However, recent developments in cobot technologies have brought in affordable, flexible, and safe torquecontrolled robot arms, which can be programmed to generate force feedback to mimic the operation of a haptic device. In this study, we use two Franka Emika Panda robot arms as a twin master-slave system to enable haptic-guided teleoperation. We propose a two layer mechanism to implement force feedback due to 1) object interactions in the slave workspace, and 2) virtual forces, e.g. those that can repel from static obstacles in the remote environment or provide task-related guidance forces. We present two different approaches for force rendering and conduct an experimental study to evaluate the performance and usability of these approaches in comparison to teleoperation without haptic guidance. Our results indicate that the proposed joint torque coupling method for rendering task forces improves energy requirements during haptic guided telemanipulation, providing realistic force feedback by accurately matching the slave torque readings at the master side.
\end{abstract}

Index Terms-Force feedback, haptics, haptic-guided manipulation, human-robot collaboration, robotics, telemanipulation, teleoperation

\section{INTRODUCTION}

$\mathbf{T}$ HE uptake of robotics has the promise facilitate the operation of repetitive tasks that require attention, such as welding, bolting, and inspection [1]. However, in complex and dangerous contexts, such as handling nuclear waste or disaster response, automation is not always possible due to the complexity and variety of tasks, and the unpredictable nature of potentially hazardous environments. In addition to such complexities, strict regulatory requirements in application domains, such as the nuclear industry, inhibits the wide uptake of autonomous robotic approaches; hence most activities in such extreme environments are predominantly dealt with through human teleoperation.

Such scenarios require the development of effective teleoperation systems that allow human operators to interact with the remote environment as realistically as possible. Although realistic force feedback can be realized through specialised haptic controllers, such devices are scarcely used in industry and are often much more expensive than today's affordable collaborative robots (cobots). This research aims to explore the use of two identical cobots in a teleoperation setup to enable haptic-guided shared control, where not only environmental interaction forces, but also forces for guidance or safeguarding, can be fed back to the user. To the best of our knowledge, ours is the first study to demonstrate a mechanism to merge task and guidance forces in a unique master-slave setup consisting of two identical cobot platforms (See Figure 1(a)).

This work was supported by by EPSRC ("HEAP", EP/S033718/1; "CoRSA"; 'NCNR", EP/R02572X/1).

${ }^{1}$ Lincoln Centre for Autonomous Systems, University of Lincoln, Lincoln, LN6 7TS, UK

${ }^{2}$ Bosch Center for Artificial Intelligence, Renningen, Germany

${ }^{3}$ School of Computer Science, University of Nottingham, Nottingham, NG8 $1 \mathrm{BB}, \mathrm{UK}$

17686310@students.lincoln.ac.uk,

asrinivasan@lincoln.ac.uk, gneumann@lincoln.ac.uk, akucukyilmaz@lincoln.ac.uk
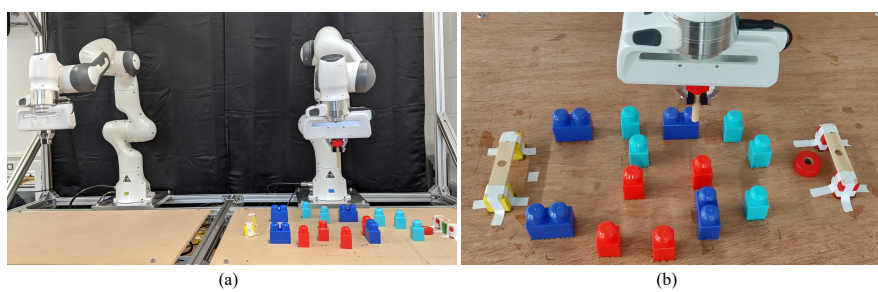

Fig. 1. (a) The teleoperation environment with the master and slave robots, (b) close-up of the telemanipulation task at the slave side.

We developed two different paradigms to transfer environmental forces acting on the slave to be felt at the master device. To evaluate the performance of these paradigms, we conducted an experimental user study to test the utility and performance of the proposed techniques in comparison to unilateral teleoperation with no force feedback. In short, the objectives of this study are:

- To develop feedback mechanisms in a master-slave teleoperation system, consisting of two 7-DoF cobot arms so that we generate the forces detected on the slave system as closely as possible at the master side as an effort to enable ideal transparency [2].

- To enable haptic-guided teleoperation for cobots by generating virtual guidance forces as well as task forces due to slave dynamics and environment interaction.

- To create an architecture that separates task and guidance forces to allow shared control.

- To test the feedback mechanisms through an experimental study with human subjects, in terms of perceived task load as well as quantitative measures of task performance and operation.

This study does not address the question of stability as the experiments were conducted with parameters that provided stable operation.

\section{RELATED WORK}

Haptic guidance is a technique aiming at improving human operation by imposing certain physical restrictions or guides on the human motion. A popular haptic guidance scheme is virtual fixtures [3], that introduces virtual overlays to guide or restrict the motion, akin to a ruler, to constrain a user's motion on a desired trajectory. Virtual fixtures were shown to enhance the execution time, quality and precision of teleoperation tasks [4]-[6]. However, there have been arguments against using virtual fixtures for training purposes, as detrimental effects of continuous robot assistance are observed [7] in line with the "guidance hypothesis" [8].

In contrast to virtual fixtures, which impose task and trajectoryrelated constraints in Cartesian space, shared control stands as an advanced robot-mediated guidance paradigm, where the human and the robot congruently perform a physically collaborative task [9]. Through sharing the control of a task, the human can become more active and effectively benefit from guidance provided when and as necessary [10]. Shared control enables predictive and progressive mechanisms to change the guidance policy [11]-[13], or allows dynamic role arbitration and variable autonomy to alter the robot's contribution to effort sharing during the task [14]-[20]. The shared control paradigm has been used to enable haptic-guided teleoperation for facilitating specific task requirements, such as post-grasp 
manipulative actions [21] or non-holonomic cutting constraints [22]. Alternatively, several researchers focused on conveying haptic cues to improve task operability when dealing with slave systems with complex morphologies [23], when controlling multiple slaves [24] or for multi-master-single-slave teleoperation [25] systems.

In teleoperation, haptic feedback is not only important to enable guidance, but also to aid human teleoperation capabilities through feeling the remote environment. As put by Powell and O'Malley [26], two types of forces are generated when implementing haptic guidance within a physical environment: 1) guidance forces arising from a perceptual overlay or an expert's operation, and 2) task forces due to interactions with the environment. Since commonly used proxy-based haptic rendering models [27] cannot distinguish between these forces, it is not straightforward to separate the effects of guidance forces from task forces when implementing haptic guided teleoperation on a single master device. As a result, most related studies focus on a single aspect of the task when generating force feedback. An exception is by Abi-Farraj et al., who combined haptic cues to provide trajectory guidance to reach good grasp configurations in addition to imposing kinematic constraints [28]. Even though this study is interesting to see a combination of different force cues, the kinematic constraints were conveyed not through kinaesthetic guidance but through vibrations. This is similar to the use of cutaneous feedback to separate task and guidance forces as proposed in [29].

The current study presents an architecture that separately generates guidance and task forces and merges them through the haptic negotiation paradigm [30]. Haptic negotiation was originally proposed to enable shared control between a human and a robot. The idea behind the paradigm is to create a set of interconnected spring-damper-mass systems to combine the operation of a human (e.g. controlling the master device) and a robot controller (e.g. generating virtual guidance forces), while interacting with a dynamic physical task (e.g. feeling the real task forces). Through controlling the gains, the robot and the human can be assigned different roles during the task, denoting dominance levels. In addition, the assistance can be completely turned on and off to trade control of the task between agents.

A similar model was later proposed by Powell and O'Malley et al. under the name shared-control proxy model [26]. Both studies integrated task and guidance forces in haptics-enabled virtual worlds, whereas the haptic negotiation model was later implemented for physical human-robot collaboration [20] and for assistive robotics [31]. The current study is the first to integrate guidance and task forces within a shared control paradigm for a teleoperation study. The two layer architecture proposed in this study closely resembles the haptic negotiation framework. Although this was not done in the current study, an advantage of this model is that it allows to assign "weights" to different channels to control how the user shall receive the force feedback from different sources. In essence, this is possible through changing the relative weight of real and virtual torque components.

\section{DUAL-ARM TELEOPERATION SETUP}

We use two Franka Emika Panda robot arms in a master-slave configuration as shown in Figure 1. Panda arms are 7-DoF robots, designed for safe human-robot collaboration. The motivation of this study is to enable haptic-guided telemanipulation by programming the master arm as a haptic interface, displaying both environmental interaction forces acting on the slave (real) and artificial corrective forces implemented for safeguarding (virtual).

Panda arms are equipped with integrated torque sensors at each joint actuator. Each robot is connected to a Franka Control Interface (FCI) on a dedicated workstation using a static ethernet connection to minimise time delays for the purposes of this study. FCI communicates with the workstation on a low-level bidirectional connection, operating at a frequency of $1 \mathrm{KHz}$. The robots are programmed using the open source libfranka library which allows the robot to be controlled through: 1) joint torque commands (gravity and joint friction compensation are handled by FCI), 2) joint positions and velocities, 3) Cartesian poses and velocities. In addition, the Simulation Lab (SL) robotics simulator and real time control engine [32] runs on both master and slave systems to issue real time control signals at $1 \mathrm{KHz}$, thus enabling torque control. A control script running on the master system provides a single point of execution and control.

\section{HAPTICS-ENABLED TELEOPERATION IMPLEMENTATION}

Most haptic systems use specialized haptic interfaces and implement virtual coupling within the task frame to match the endeffector positions and generate force feedback [33]-[35]. However, working with identical master/slave arms brings different constraints due to robot morphology as well possibilities when generating force feedback due to the joint sensing capabilities. In particular, when designing master/slave teleoperation with twin arms, we are not only concerned with end-effector positions, but rather want to match all slave joints to the exact movement of the whole master arm, which can, for example, be beneficial for obstacle avoidance. Such joint coupling can be achieved through:

- Joint angle coupling: matching the position of the joints and generating force feedback using bilateral position control between devices (this is similar to how forces can be rendered through virtual coupling with an impedance-type haptic interface).

- Joint torque coupling: matching the torques sensed at the joints.

In this study, we implemented both techniques to render real task forces at the master side. In addition, virtual forces are generated using repulsive potential fields around obstacles. Both virtual (repulsion) and real (task) forces are integrated to be displayed as the commanded torque at the master side, following the haptic negotiation framework [30] using equal weights for the components.

\section{A. Master to Slave Control with No Feedback}

As the baseline setup, we implemented a position-based unilateral PD controller to control the slave motion via the master. This baseline implementation realizes teleoperation to move the slave arm, however, no feedback is provided at the master side. For this reason, we call this baseline the "No Feedback" condition. The torque command at the slave side is computed using a PD controller with gains $\mathbf{k}_{p}=[240,240,240,240,100,60,20]^{\top}$ and $\mathbf{k}_{d}=[20,20,20,20,12,10,6]^{\top}$.

\section{B. Artificial Force Rendering}

We implemented virtual repulsive fields around obstacles to demonstrate how artificial force rendering can be integrated in the architecture. The repulsive fields are used to push the robot end-effector away from obstacles if a certain distance threshold is breached, and is converted into the resulting joint torques as

$$
\boldsymbol{\tau}_{v}=\mathbf{J}^{T} \frac{k \sum_{i=1}^{N}\left(\mathbf{x}_{r}-\mathbf{o}_{i}\right)}{\left\|\sum_{i=1}^{N}\left(\mathbf{x}_{r}-\mathbf{o}_{i}\right)\right\|} \quad \text { if }\left\|\left(\mathbf{x}_{r}-\mathbf{o}_{i}\right)\right\|<d,
$$

where $\boldsymbol{\tau}_{v}$ is the joint torques due to virtual guides, $\mathbf{J}$ is the Jacobian for the current robot joint configuration and the term to the right of $\mathbf{J}^{T}$ acts as a virtual external force acting on the end-effector. In this external force computation, $k$ stands as the gain, $\mathbf{x}_{r}$ and $\mathbf{o}_{i}$ are the 3D Cartesian space position of the slave end-effector and the $i^{t h}$ obstacle respectively. 


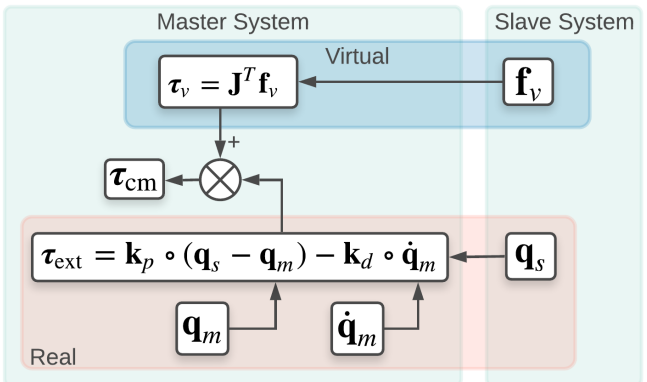

Fig. 2. Force feedback architecture based on joint angle coupling. A PD controller gives the "real" force feedback component (pink) while "virtual" guidance component is shown in blue.

\section{Force Feedback using Joint Angle Coupling}

This technique implements bilateral position-position teleoperation using a PD controller to get the arms to mirror one another's motion. Joint torques due to slave dynamics and environmental contact at the slave side are calculated as seen in Figure 2 in the lower segment in pink, labelled Real. Artificial forces are generated as shown in the upper blue segment labelled Virtual. In the figure, $\mathbf{f}_{v}$ denotes the virtual/guidance force to be delivered to the master robot end-effector, $\mathbf{q}_{s}$ and $\mathbf{q}_{m}$ are slave and master joint angles respectively, $\mathbf{M}(\mathbf{q})$ is the mass matrix and $\mathbf{k}_{p}, \mathbf{k}_{d}$ are controller gains. $\boldsymbol{\tau}_{\mathrm{cm}}$ is the resulting master torque command, computed as the equally weighted sum of virtual and real torques.

The forward dynamics equation for a robot manipulator is

$$
\boldsymbol{\tau}=\mathbf{M}(\mathbf{q}) \ddot{\mathbf{q}}+\mathbf{C}(\mathbf{q}, \dot{\mathbf{q}}) \mathbf{q}+\mathbf{g}(\mathbf{q})+\tau_{\mathrm{ext}},
$$

where $\mathbf{M}(\mathbf{q})$ is the mass matrix for joint configuration $\mathbf{q}, \mathbf{C}(\mathbf{q}, \dot{\mathbf{q}})$ is the Coriolis term and $\mathbf{g}(\mathbf{q})$ gives the joint torques due to gravity.

The robot arms used for this project are gravity compensated so the robot dynamics equation for the control loop does not include the term $\mathbf{g}(\mathbf{q})$. As the teleoperation task involved low velocities, the Coriolis term $\mathbf{C}(\mathbf{q}, \dot{\mathbf{q}}) \mathbf{q}$ also becomes irrelevant and is ignored. A simple PD controller for force feedback is defined as

$$
\tau_{\text {ext }}=\mathbf{k}_{p} \circ\left(\mathbf{q}_{s}(t)-\mathbf{q}_{m}(t)\right)-\mathbf{k}_{d} \circ \dot{\mathbf{q}}_{m}(t),
$$

where $\circ$ is the element-wise Hadamard product, $\boldsymbol{\tau}_{\text {ext }}$ is the torque control command due to contact forces for the master, $\mathbf{q}_{m}(t)$ and $\mathbf{q}_{s}(t)$ are respectively the master and the slave joint configurations at time step $t, \dot{\mathbf{q}}_{m}$ is the master joint velocity, and $\mathbf{k}_{p}=[120,120,120,120,20,20,4]^{\top}$ and $\mathbf{k}_{d}=$ $[7.5,7.5,7.5,7.5,2.25,2.25,0.75]^{\top}$ are respectively the proportional and derivative gains, which are tuned to enable stable teleoperation of the arms.

\section{Force Feedback using Joint Torque Coupling}

Figure 3 shows the torque based force feedback control architecture, where $\boldsymbol{\tau}_{s}$ is the slave joint torque sensor data, $\mathbf{g}(\mathbf{q})$ is the gravity acting on the joints and $\mathbf{k}_{\tau}=[0.9,0.9,0.45,0.9,0.45,0.9,0.9]^{\top}$ is the feedback scaling factor. Note that this architecture can only be applied on systems where the master and slave robots are identical and employed with force/torque sensors at the joints. An additional step of inverse and forward statics is needed when working with robots that have different morphology, as shown in Figure 4.

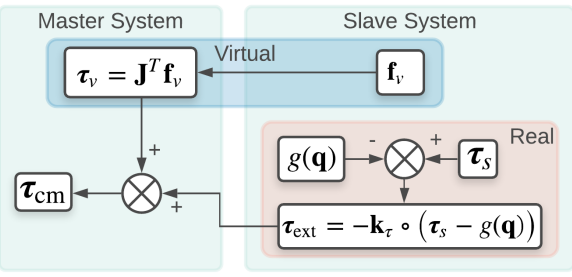

Fig. 3. Force feedback architecture based on joint torque coupling. Slave joint torque sensors are applied on the master after eliminating gravity-induced torque.

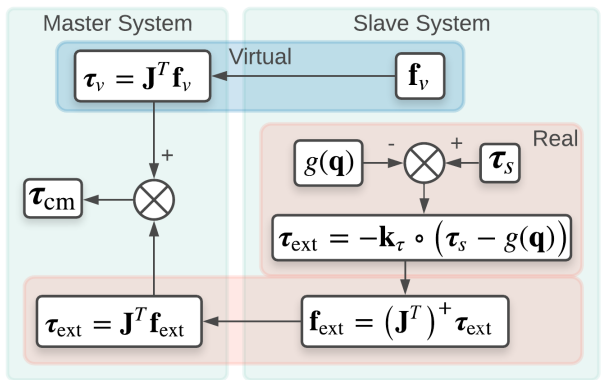

Fig. 4. Force feedback architecture based on joint torque coupling for different master/slave morphologies achieved by converting force-feedback torque $\left(\boldsymbol{\tau}_{\text {ext }}\right)$ to a task space force $\left(\boldsymbol{f}_{\text {ext }}\right)$.

\section{E. Dealing with Backdrivability}

Both position-position and force-position control systems, when implemented naively, produce low torques $(<1 \mathrm{Nm})$ that oppose the free motion of the master robot even without any external forces on the slave system resulting in a sluggish system that is difficult to operate. This behavior is generally expected when working with noisy data from a real sensor. Some of this noise can be eliminated using specially tuned filters. For eliminating the noise in the torque sensors, we use a fast and efficient Kalman filter, which works well with Gaussian noise. In the prediction step, estimates of the current state are computed as $\mathbf{x}_{\mathbf{t}+\mathbf{1}}^{-}=\mathbf{F} \mathbf{x}_{\mathbf{t}}^{+}$, where $\mathbf{x}_{\mathbf{t}}$ is the $14 \times 1$ state vector $\left[\begin{array}{ll}\boldsymbol{\tau} & \dot{\boldsymbol{\tau}}^{\top}\end{array}\right]^{\top}$ and $\mathbf{F}$ is the $14 \times 14$ state-transition model $\left[\begin{array}{l}\mathbf{I} \operatorname{diag}(\Delta t) \\ 0\end{array}\right]$ $(\Delta t=0.001)$. The notation $\vec{x}_{t}^{-}$and $\vec{x}_{t}^{+}$discriminates between prior and posterior estimates of the mean (the same notation is used for the covariance). The $14 \times 14$ covariance estimate is computed as

$$
\mathbf{P}_{t+1}^{-}=\mathbf{F} \mathbf{P}_{\mathbf{t}}^{+} \mathbf{F}^{\top}+\mathbf{Q}
$$

where $\mathbf{Q}$ is the $14 \times 14$ process noise covariance, which was estimated using a short sample of the sensor data.

The update step uses new observations to improve the estimated state and estimate covariance as

$$
\begin{aligned}
\mathbf{x}_{t}^{+} & =\mathbf{x}_{t}^{-}+\mathbf{K}_{t}\left(\mathbf{z}_{t}-\mathbf{H} \mathbf{x}_{t}^{-}\right), \\
\mathbf{P}_{t}^{+} & =\mathbf{P}_{t}^{-}-\mathbf{K}_{t} \mathbf{S}_{t} \mathbf{K}_{t}^{\top}
\end{aligned}
$$

respectively, where $\mathbf{K}$ is the $14 \times 7$ Kalman gain, $\mathbf{z}_{\mathbf{t}}$ is the $7 \times 1$ observation vector and $\mathbf{H}$ is the $7 \times 14$ observation model. The innovation covariance $\mathbf{S}_{\mathbf{t}}(7 \times 7)$, where innovation denotes the error between the prediction and observation, is computed as $\mathbf{S}_{\mathbf{t}}=\mathbf{H} \mathbf{P}_{t}^{-} \mathbf{H}^{\top}+\mathbf{R}$. Here, $\mathbf{R}$ is the $7 \times 7$ observation noise covariance, whereas the Kalman gain is computed as $\mathbf{K}_{t}=\mathbf{P}_{t}^{-} \mathbf{H} \mathbf{S}_{t}^{-1}$.

After Kalman filtering, we still observed some undesirable torque values $\mathbf{e}(\mathbf{q})$, which change depending on the joint configurations. These may be an artefact of a robust control running on the robot to correct for an imprecise gravity compensation. Such an error cannot 
be reliably eliminated without learning the robot dynamics, which is beyond the scope of this work. It is therefore desirable to ignore values below a certain threshold. Yet, a simple step function may result in sudden jerks (as the torque value jumps across the threshold boundary). Hence, we used a continuous conditional cubic function to squash the values within a threshold, while maintaining a linear output for everything else, where $\tau_{m}$ are the measured torques and $\tau_{\mathrm{sq}}$ are the computed squashed torques

$$
\boldsymbol{\tau}_{\mathrm{sq}}= \begin{cases}\boldsymbol{\tau}_{m}^{3}, & \text { if }-1<\boldsymbol{\tau}_{m}<1 \\ \boldsymbol{\tau}_{m}, & \text { otherwise }\end{cases}
$$

\section{EXPERIMENTAL STUdY}

In order to measure and compare the performance of human operators, we conducted a user study in a task consisting of gross and fine manipulation segments as shown in Figure 1(b). We measured task completion times and physical effort, as well as the perceived physical and mental workload during the task. We followed the University of Lincoln ethical guidelines and obtained ethical approval before the commencement of the studies. The subjects signed an informed consent form explaining privacy and safety information as well as risks and benefits during the experiment. They were informed that they were free to quit the study at any time or retract their data after the experiment.

At the beginning of the trials, the participants were provided with an instruction document explaining the details of the experiment and their role as a robot operator. They were then given a demonstration of the task and had a practice session to operate the robot before the experiment. Appropriate automatic and manual safety measures were in place including physical kill switches moderated by the experimenter to stop the robots in case of emergency.

In the experiment, the master and the slave system were mounted in the same room, enabling clear visual observation of the slave robot and the task environment while manipulating the master. 2-fingered Franka grippers were attached to both the master and slave arms. A peg was rigidly gripped by the slave robot, hence the user was not required to open or close the grippers during the task.

The experimental task is depicted in Figure 5. The task started with the slave peg inserted into the start hole. The subjects were asked to remove the peg from the hole and insert it into the puck. Then, they were asked to slide the pegged puck across the surface while steering around obstacles. At the end of the task, they were asked to remove the peg from the puck and insert it into the fixed finish hole.

This manipulation sequence involved two kinds of operational modes, namely fine and gross manipulation (See Figure 6). The experimental workspace was divided into two main regions of interest

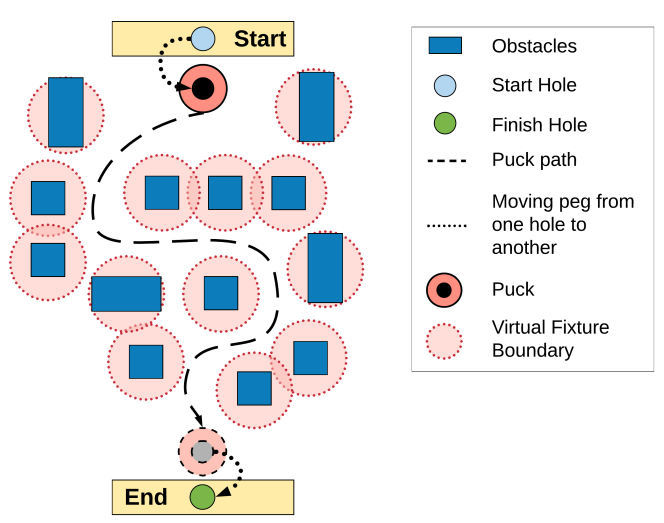

Fig. 5. A bird's eye view of the experiment task at the slave side.

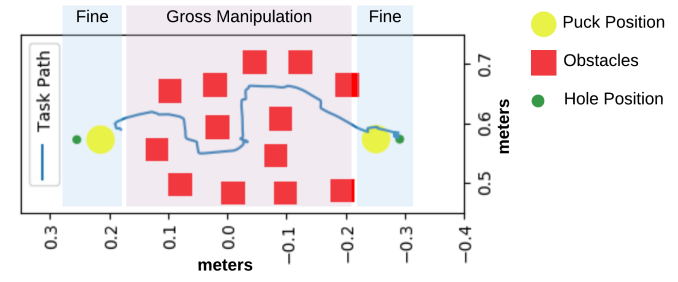

Fig. 6. The path taken by a subject is plotted with obstacles in red and the initial and final positions in green. Yellow circles mark the start and end positions for the puck.

based on the kind of manipulation skills employed as can be seen in Figure 6. The fine manipulation regions involved the peg-in-thehole operations, which require slow, precise $6 \mathrm{DoF}$ control. The gross manipulation region required manoeuvring and involved pushing an object against the surface.

The experiment consisted of three feedback conditions:

1) No Feedback: Unilateral teleoperation is implemented with no real or virtual haptic feedback at the master side

2) Position: Joint angle coupling and virtual haptic feedback was displayed at the master side

3) Torque: Joint torque coupling and virtual haptic feedback was displayed at the master side

We followed a within-subjects design, where subjects experimented with all three conditions. The experiment took up approximately 30 minutes. Under each condition, subjects completed 3 trials, going forward and backward in between start and finish holes with the slave arm using the master interface. At the end of each trial, the subjects released the master arm, and the robots were reset to a predefined neutral position. This was done to ensure the same starting joint configuration for each trial, and in order to avoid the robot joints to end up in poor configurations or near singularities, which would affect the manoeuvrability.

In order to avoid ordering and carryover effects, the order of trials was randomised using a Latin square design. 6 participants ( 4 female, 2 male) aged between 19 and 26 participated in this study. The subjects were randomly assigned to groups using a computerised random number generator. The right or left hand dominance of the participants was not considered, as the task requires both hands to simultaneously manipulate the master arm. According to the literature [36], the grasping and placing behaviour of right and left handed groups in bi-manual tasks is similar, so it can be safely ignored for this experiment. It was noted that the sound due to the physical contact of the slave robot with the environment was audible and reflected as an acoustic cue, which could affect the experiment results. To control for possible bias, the participants wore noise-cancelling head gear.

Throughout the task, timestamped joint configurations $\mathbf{q}$, joint velocities $\dot{\mathbf{q}}$, joint torques $\boldsymbol{\tau}$, and Cartesian position for the endeffector $\mathbf{p}$ are collected for both robots. Measures used in the study are as follows:

1) Force feedback realism: The error between the joint torques on the master and slave robots are used as a measure of haptic teleoperation quality to enable transparency.

2) Task performance: As a primary measure of task performance, we recorded the task completion time for individual trials.

3) Energy: The total energy spent during a task is computed by integrating the human exerted power over time as

$$
E=\sum_{t=0}^{T} \boldsymbol{\tau}(t) \cdot \dot{\mathbf{q}}(t),
$$

where $\mathrm{T}$ is the duration of the trial. 


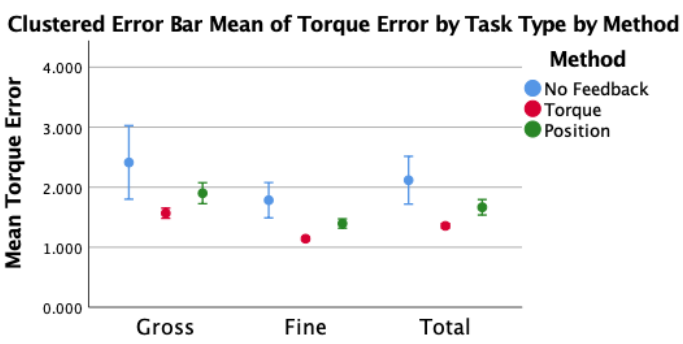

Fig. 7. The mean difference between the measured torques from slave and master robot joint sensors. Error bars denote $\pm 2 \mathrm{SE}$.

4) Task load: At the end of each condition block, the participants were given the NASA Task Load Index (TLX), consisting of 6 questions on a 21 point scale, to record the perceived physical and mental workload during the task.

\section{RESULTS AND DISCUSSION}

During the experiment, a total of 54 trials were conducted. However, the logger failed to save the collected data for 2 trials, therefore the total number of available trials is $N=52$. The total number of questionnaire responses recorded is $N=18$ (one per participant per condition).

Figure 7 shows the mean torque error for different methods. A two-way ANOVA indicates significant main effects of the feedback condition $(F(2,98)=13.865, p<0.001)$ and the manipulation type (fine vs. gross) $(F(1,98)=19.915, p<0.001)$. No significant interaction effects are observed. Due to large differences in the variances of groups, we conducted Games-Howell nonparametric post-hoc tests to examine the pairwise differences. The tests indicate statistically significant differences between joint-angle coupling and No Feedback conditions for gross manipulation $(p=0.268)$. However, statistically significant differences between all other pairs of control techniques are observed. As expected, the highest torque error is observed under the No Feedback condition, whereas the lowest is observed for jointtorque coupling. This indicates that more realistic task forces can be rendered using the joint-torque coupling technique.

Figure 8 shows the mean completion times of each trial and the standard errors of the means. Two-way ANOVA indicate no significant main effects of feedback condition $(F(2,98)=0.748$, $p=0.476)$ or the manipulation type $(F(1,98)=2.211, p=0.140)$.

Figure 9 shows the mean energy spent across feedback conditions and manipulation type. Two-way ANOVA indicates a significant effect of the feedback condition $(F(2,98)=7.014, p<0.005)$. A significant effect is observed also for manipulation type $(F(2,98)=$ 208.765, $p<0.001$ ), indicating lower energy expenditure under fine manipulation. Bonferroni-corrected post-hoc tests indicate that joint torque coupling requires significantly less energy than both nofeedback $(p<0.05)$ and joint angle coupling $(p<0.005)$.

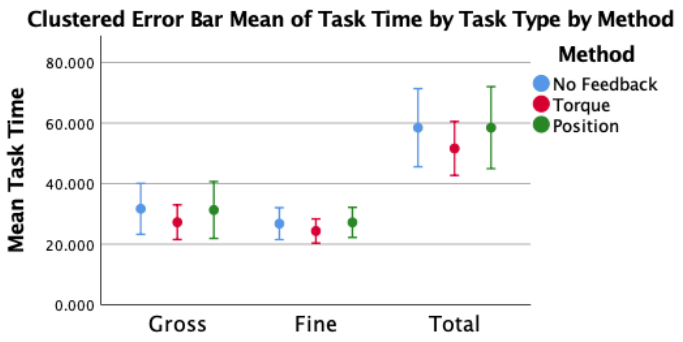

Fig. 8. The average time taken to complete the tasks by manipulation type and feedback method. Error bars denote \pm 2 SE.

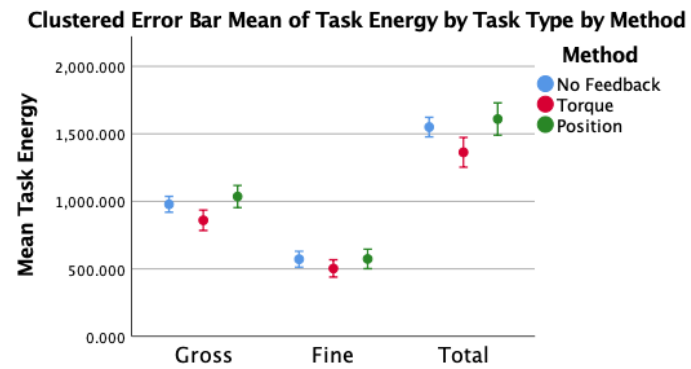

Fig. 9. The energy spent to complete the task for each task type and feedback method. Error bars denote \pm 2 SE.

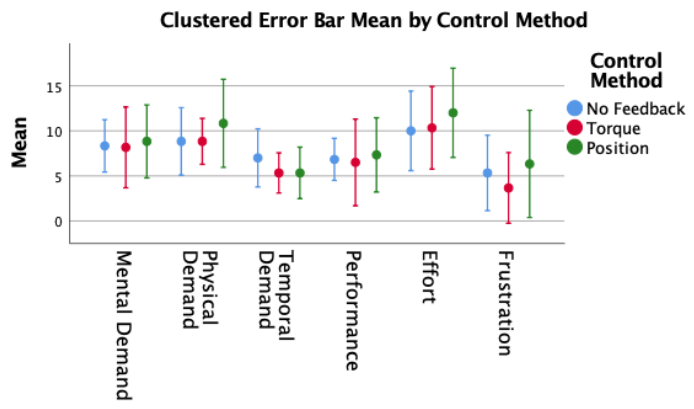

Fig. 10. Raw NASA-TLX scores for perceived task workload (lower values are better, including for performance).

Finally, the participants' NASA TLX scores are shown in Figure 10. Statistical analysis showed no significant difference between different feedback methods, indicating that the subjects did not feel any of the conditions as being more demanding than the others.

\section{CONCLUSIONS AND FUture WORK}

In this study, we developed two methods for enabling force feedback when teleoperating a 7-DoF robotic arm using an identical master interface. Our methods were used to display environmental forces acting on the slave arm. Task-related forces are integrated with artificial force cues for guidance. An experimental task was designed to involve both fine and gross manipulation when comparing the haptic feedback generation mechanisms.

Experimental analysis of the implemented guidance and force feedback techniques indicate no significant difference between the task completion times for the different methods. Similarly, questionnaire results showed no significant differences in terms of mental or physical task load. However, we observed that the joint torque coupling method generates more realistic task forces, enabling better transparency by matching the slave torque readings at the master side. Also, it requires less energy to complete the task for both fine and gross manipulation operations. In addition, we observed in the experiments that joint angle coupling method is more sensitive to time delays, causing sluggishness and viscosity in motion as the joint angle differences grows.

This study was completed with a limited number of participants. However, the existence of statistically significant differences even with this small population is promising to illustrate the benefit of the proposed method. We did not observe any differences in subjects' perception of the task load. We believe this could be due to the simplicity of the task. Future experiments are planned to make the task more difficult by separating the slave and master workspace to avoid direct visibility of the task space. In future work, we will study more sophisticated haptic guidance cues that allow variable autonomy and trajectory control. 
In this study, guidance forces were generated around static obstacles, and the implemented technique does not take the shape of the obstacles into account. In future work, we will explore the use of computer vision to enable automatic scene understanding and dynamic object recognition to provide guidance cues accordingly. In addition, in this study, we used the data coming from slave sensors to generate environmental forces, and leverage task information to enable open-loop virtual fixtures for guidance generation. However, the proposed architecture allows different ways for virtual guidance forces to be implemented.

This work is an effort to integrate task and guidance forces over a single master interface. The implemented concept has been studied in [26] as Gross Assistance. Unfortunately, this mechanism can encounter situations, in which haptic feedback and guidance forces annihilate each other creating misleading sensory feedback. Alternative ways of integrating different forces will be investigated in future studies. For instance, using the haptic negotiation framework, we intend to implement spatially and temporally separate guidance and task forces as well as weighting force channels depending on task requirements and user needs.

\section{REFERENCES}

[1] D. W. Seward and M. J. Bakari, "The use of robotics and automation in nuclear decommissioning," in 22nd International Symposium on Automation and Robotics in Construction ISARC, 2005, pp. 11-14.

[2] Y. Yokokohji and T. Yoshikawa, "Bilateral control of master-slave manipulators for ideal kinesthetic coupling-formulation and experiment," IEEE Transactions on Robotics and Automation, vol. 10, no. 5, pp. 605620, 1994

[3] L. B. Rosenberg, "Virtual fixtures: perceptual tools for telerobotic manipulation," 1993 IEEE Annual Virtual Reality International Symposium, pp. 76-82, 1993.

[4] P. Marayong, M. Li, A. M. Okamura, and G. D. Hager, "Spatial motion constraints: Theory and demonstrations for robot guidance using virtual fixtures," in 2003 IEEE International Conference on Robotics and Automation, vol. 2. IEEE, 2003, pp. 1954-1959.

[5] A. B. Kuang, S. Payandeh, B. Zheng, F. Henigman, and C. L. MacKenzie, "Assembling virtual fixtures for guidance in training environments," in 12th Intl. Symposium on Haptic Interfaces for Virtual Environment and Teleoperator Systems, HAPTICS'04. IEEE, 2004, pp. 367-374.

[6] M. Selvaggio, G. Notomista, F. Chen, B. Gao, F. Trapani, and D. Caldwell, "Enhancing bilateral teleoperation using camera-based online virtual fixtures generation," in 2016 IEEE/RSJ International Conference on Intelligent Robots and Systems (IROS). IEEE, 2016, pp. 1483-1488.

[7] L. M. Crespo and D. J. Reinkensmeyer, "Effect of robotic guidance on motor learning of a timing task," in 2008 2nd IEEE RAS \& EMBS International Conference on Biomedical Robotics and Biomechatronics. IEEE, 2008, pp. 199-204.

[8] R. A. Schmidt, "Frequent augmented feedback can degrade learning: Evidence and interpretations," in Tutorials in motor neuroscience. Springer, 1991, pp. 59-75.

[9] D. A. Abbink, T. Carlson, M. Mulder, J. C. de Winter, F. Aminravan, T. L. Gibo, and E. R. Boer, "A topology of shared control systems-finding common ground in diversity," IEEE Transactions on Human-Machine Systems, vol. 48, no. 5, pp. 509-525, 2018.

[10] M. K. O'Malley, A. Gupta, M. Gen, and Y. Li, "Shared control in haptic systems for performance enhancement and training," Journal of Dynamic Systems, Measurement, and Control, vol. 128, no. 1, pp. 75-85, 2006.

[11] B. A. C. Forsyth and K. E. MacLean, "Predictive haptic guidance: Intelligent user assistance for the control of dynamic tasks," IEEE Transactions on Visualization and Computer Graphics, vol. 12, no. 1, pp. 103-113, 2006.

[12] J. Huegel and M. O'Malley, "Progressive haptic and visual guidance for training in a virtual dynamic task," in Haptics Symposium, 2010 IEEE, mar. 2010, pp. 343-350.

[13] J. Lee and S. Choi, "Effects of haptic guidance and disturbance on motor learning: Potential advantage of haptic disturbance," in Haptics Symposium, 2010 IEEE, march 2010, pp. 335-342.

[14] D. P. Losey, C. G. McDonald, E. Battaglia, and M. K. O’Malley, “A review of intent detection, arbitration, and communication aspects of shared control for physical human-robot interaction," Applied Mechanics Reviews, vol. 70, no. 1, p. 010804, 2018.
[15] A. Kucukyilmaz, S. O. Oguz, T. M. Sezgin, and C. Basdogan, "Improving human-computer cooperation through haptic role exchange and negotiation," in Immersive Multimodal Interactive Presence. Springer, 2012, pp. 229-254.

[16] A. Kucukyilmaz, T. Sezgin, and C. Basdogan, "Intention recognition for dynamic role exchange in haptic collaboration," IEEE Transactions on Haptics, vol. 6, no. 1, pp. 58-68, 2013.

[17] D. A. Abbink, M. Mulder, and E. R. Boer, "Haptic shared control: smoothly shifting control authority?" Cognition, Technology \& Work, vol. 14, no. 1, pp. 19-28, 2012.

[18] Y. Li, K. P. Tee, W. L. Chan, R. Yan, Y. Chua, and D. K. Limbu, "Continuous role adaptation for human-robot shared control," IEEE Transactions on Robotics, vol. 31, no. 3, pp. 672-681, 2015.

[19] A. D. Dragan and S. S. Srinivasa, "A policy-blending formalism for shared control," The International Journal of Robotics Research, vol. 32, no. 7, pp. 790-805, 2013

[20] A. Mörtl, M. Lawitzky, A. Kucukyilmaz, T. M. Sezgin, C. Basdogan, and S. Hirche, "The role of roles: Physical cooperation between humans and robots." Int. J. Robotic Res., vol. 31, no. 13, pp. 1656-1674, 2012.

[21] E. A. M. Ghalamzan, F. Abi-Farraj, P. R. Giordano, and R. Stolkin, "Human-in-the-loop optimisation: mixed initiative grasping for optimally facilitating post-grasp manipulative actions," in 2017 IEEE/RSJ International Conference on Intelligent Robots and Systems (IROS). IEEE, 2017, pp. 3386-3393.

[22] R. Rahal, F. Abi-Farraj, P. Giordano, and C. Pacchierotti, "Haptic sharedcontrol methods for robotic cutting under nonholonomic constraints," in 2019 IEEE/RSJ International Conference on Intelligent Robots and Systems (IROS), 2019.

[23] F. Abi-Farrajl, B. Henze, A. Werner, M. Panzirsch, C. Ott, and M. A. Roa, "Humanoid teleoperation using task-relevant haptic feedback," in 2018 IEEE/RSJ International Conference on Intelligent Robots and Systems (IROS). IEEE, 2018, pp. 5010-5017.

[24] M. Selvaggio, F. Abi-Farraj, C. Pacchierotti, P. R. Giordano, and B. Siciliano, "Haptic-based shared-control methods for a dual-arm system," IEEE Robotics and Automation Letters, vol. 3, no. 4, pp. 4249-4256, 2018.

[25] M. Panzirsch, R. Balachandran, and J. Artigas, "Cartesian task allocation for cooperative, multilateral teleoperation under time delay," in 2015 IEEE International Conference on Robotics and Automation (ICRA). IEEE, 2015, pp. 312-317.

[26] D. Powell and M. K. O'Malley, "Efficacy of shared-control guidance paradigms for robot-mediated training," in 2011 IEEE World Haptics Conference. IEEE, 2011, pp. 427-432.

[27] C. B. Zilles and J. K. Salisbury, "A constraint-based god-object method for haptic display," in Proceedings 1995 IEEE/RSJ International Conference on Intelligent Robots and Systems. Human Robot Interaction and Cooperative Robots, vol. 3. IEEE, 1995, pp. 146-151.

[28] F. Abi-Farraj, C. Pacchierotti, O. Arenz, G. Neumann, and P. R. Giordano, "A haptic shared-control architecture for guided multi-target robotic grasping," IEEE transactions on haptics, 2019.

[29] E. Pezent, S. Fani, J. Clark, M. Bianchi, and M. K. O’Malley, "Spatially separating haptic guidance from task dynamics through wearable devices," IEEE transactions on haptics, 2019.

[30] S. O. Oguz, A. Kucukyilmaz, T. M. Sezgin, and C. Basdogan, "Haptic negotiation and role exchange for collaboration in virtual environments," in 2010 IEEE Haptics Symposium. IEEE, 3 2010, pp. 371-378. [Online]. Available: https://doi.org/10.1109/HAPTIC.2010.5444628

[31] A. Kucukyilmaz and Y. Demiris, "Learning shared control by demonstration for personalized wheelchair assistance," IEEE transactions on haptics, vol. 11, no. 3, pp. 431-442, 2018.

[32] S. Schaal, "The SL Simulation and Real-Time Control Software Package," University of Southern California, Tech. Rep., 2006. [Online]. Available: http://www-clmc.usc.edu/publications/S/schaal-TRSL.pdf

[33] R. J. Adams and B. Hannaford, "Stable haptic interaction with virtual environments," IEEE Transactions on Robotics and Automation, vol. 15, no. 3, pp. 465-474, 1999.

[34] G. Sankaranarayanan and B. Hannaford, "Experimental internet haptic collaboration using virtual coupling schemes," in 2008 Symposium on Haptic Interfaces for Virtual Environment and Teleoperator Systems. IEEE, 2008, pp. 259-266.

[35] M. Kim and D. Lee, "Improving transparency of virtual coupling for haptic interaction with human force observer," Robotica, vol. 35, no. 2, pp. 354-369, 2017.

[36] C. M. Hughes, P. Reißig, and C. Seegelke, "Motor planning and execution in left- and right-handed individuals during a bimanual grasping and placing task," Acta Psychologica, vol. 138, no. 1, pp. 111-118, 2011. 